\title{
Medical Image of the Week: Bronchial Clot Removal via Cryotherapy
}

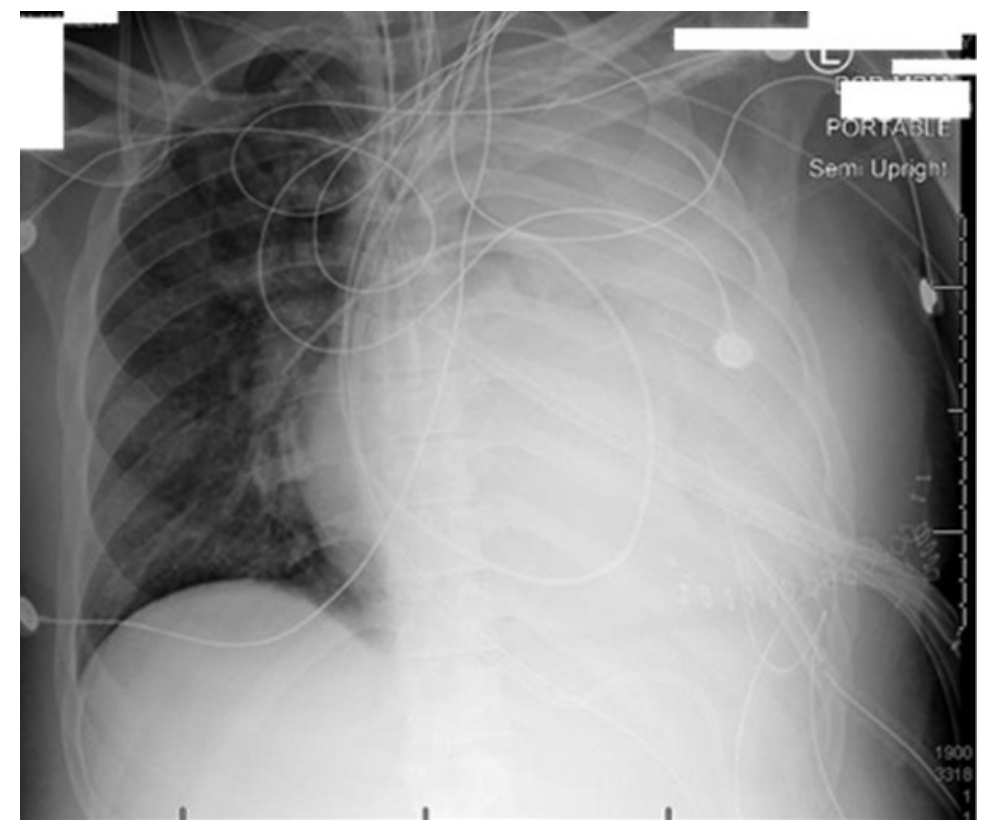

Figure 1. Chest x-ray showing complete opacification of the left hemithorax.

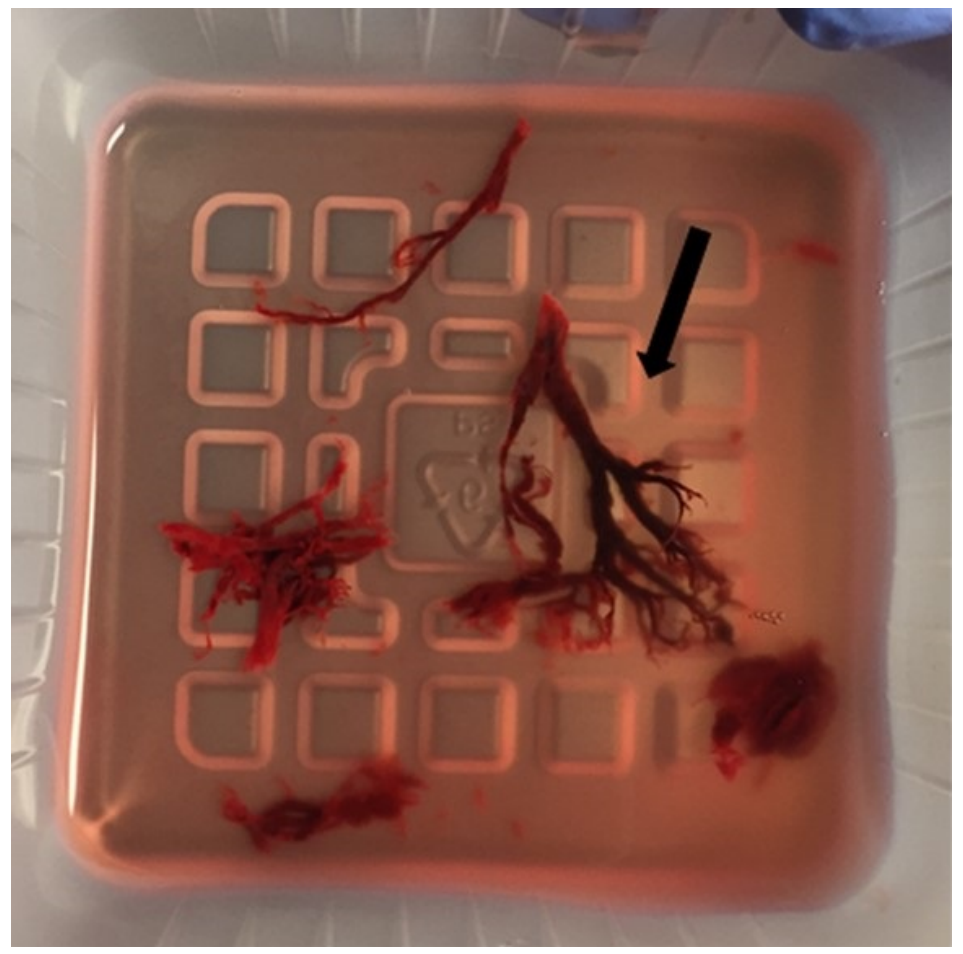

Figure 2. Flexible bronchoscopy with cryotherapy was used to remove clot that formed casts of the bronchial tree. Black arrow: depicts segmental branch of the left upper lobe. 
A 38-year-old man with a history of non-ischemic dilated cardiomyopathy presented with decompensated heart failure, acute renal failure, and possible sepsis. He underwent right cardiac catheterization but developed hemoptysis with concern for pulmonary artery rupture. Subsequently, the patient suffered a cardiac arrest but was resuscitated. Emergent bronchoscopy revealed copious amounts of blood and clot that could not be cleared at the time. The patient was then taken to the operating room and placed on AA ECMO (left ventricle to aorta). The following morning chest x-ray (Figure 1) revealed a completely opacified left lung. Flexible bronchoscopy showed blood clot along the entire left bronchial tree. Initial attempts to remove the clot with suction and endobronchial graspers was unsuccessful. Ultimately, cryotherapy was used to remove the majority of the clot in fragments (Figure 2).

The use of cryotherapies and specifically, in this case, a cryoprobe, has been shown to safely and effectively remove thrombus from the bronchial tree. The basis behind this technique is the use of pressurized nitrous oxide or carbon dioxide to cool a metal probe tip. The probe then freezes any substance it comes in direct contact with, such as a blood clot. Thus, cryoadherence of the probe to the clot allows for effective removal via flexible endoscopy. Sriratanaviriyakul et al. (1) reported success rates for cryoextraction of blood clots to be $>90 \%$.

Cathy V. Ho MD, Ryan Matika MD, and Mimi Amberger MD ${ }^{1}$ Division of Trauma, Critical Care, Burn and Emergency Surgery. Department of Surgery

${ }^{2}$ The Department of Anesthesia

University of Arizona

Tucson, AZ USA

\section{Reference}

1. Sriratanaviriyakul N, Lam F, Morrissey BM, Stollenwerk N, Schivo M, Yoneda KY.Safety and clinical utility of flexible bronchoscopic cryoextraction in patients with non-neoplasm tracheobronchial obstruction: a retrospective chart review. J Bronchology Interv Pulmonol. 2015 Oct;22(4):288-93. [CrossRef] [PubMed] 